\title{
A Multi-Class Fisher Linear Discriminant Approach for the Improvement in the Accuracy of Complex Texture Discrimination
}

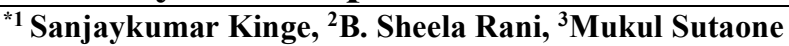 \\ ${ }^{* 1}$ Research Scholar, Sathyabama University, Chennai and Assistant Professor, Department of Electronics and \\ Telecommunication, MIT College of Engineering Pune, India \\ ${ }^{2}$ Director - Research, Sathyabama University, Chennai, (Tamil Nadu), India. \\ ${ }^{3}$ Professor and Deputy Director, College of Engineering Pune (Maharashtra), India. \\ Email: sanjaykinge100@gmail.com,directorresearch@sathyabamauniversity.ac.in,mssutaone.extc@coep.ac.in
}

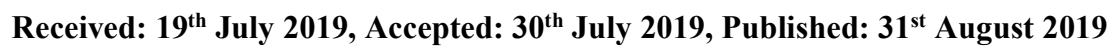

\begin{abstract}
Texture segmentation has a wide spectrum of applications in diverse fields. This paper presents an elaborated Fisher Linear Discriminant (FLD) based semi-supervised approach for improving the accuracy of segmentation of multi-class complex fine textures. Gabor filter and local statistics (local variance) are used for feature extraction of texture images. Texture segments in the image are separated using K-means clustering. The results obtained using K-means clustering are refined by multi-class Fisher Linear Discriminant (MFLD). The algorithm is tested on wide varieties of several hundred homogenous and complex textures from five texture databases viz. Outex texture database, vision texture database (Vistex), Brodatz textures, Prague textures and Pertex texture database. Fisher distance (FD) is a measure of texture separability. Segmentation of complex textures is relatively a difficult task. The improvement in the segmentation accuracy of complex textures is achieved simply by the termination of MFLD based algorithm when Fisher distance (FD) ceases to increase with the increasing iterations of MFLD. After a quantitative analysis of the experimentation, it is concluded that the segmentation accuracy of complex textures and the combination of complex and homogeneous fine textures (with small texture primitives) increases as high as $\mathbf{2 9 . 8 3 \%}$ with the increasing iterations of MFLD resulting in a significant improvement at the boundaries. Detailed results are provided in the experimentation and results section of the paper. The results achieve the second rank for 21 benchmark images among the ten state-of-the-art algorithms.
\end{abstract}

\section{Keywords}

Gabor Filter, Highly Complex Texture, Multi-Class Fisher Discriminant, Texture Database.

\section{Introduction}

Image segmentation is a very important step in image analysis and is used in diverse fields such as computer vision, pattern recognition, medical imaging, machine learning and many other fields. The widely used image segmentation methods are thresholding methods, region and edge-based techniques, clustering and watershed segmentation techniques, level set methods and parametric methods, etc [12]. Texture segmentation is one of the problem domains. This paper describes an elaborated Fisher Linear Discriminant (FLD) based semi-supervised approach for improving the accuracy of segmentation of multi-class complex textures (wherein only a number of classes is required to be known). Texture segmentation problem is difficult due to the fact that researchers have not been able to provide a universal definition of texture till date due to very wide varieties of textures existing in the nature. The popular thesaurus specifies a literal meaning of the word texture as a characteristic of a surface. Clausi DA [5] defines texture as spatial distribution of intensities in image regions observed to be homogeneous throughout the region by a normal human eye.

Texture understanding and human vision are highly related to each other. This has promoted research activities in all texture problem domains viz. texture feature extraction, texture segmentation, texture classification, texture synthesis and shape from textures [32].

Texture has over a million applications $[13,30]$ in different domains as stated previously. The spectrum of applications includes texture-based image segmentation such as an analysis of remotely sensed sea-ice images [33, 34], location of forests and agriculture lands from remotely sensed images, medical image analysis like diagnosis of diseases from X-ray image and the list goes on. Areas such as image data retrieval using texture classification, texture synthesis for computer graphics such as generation of natural scenes, animations, 3D imaging, and surface shape extractions in industry, add to the tally.

Texture segmentation is a two-step process. The first step is to extract meaningful features from an input image. Many feature extraction methods have been proposed by the researchers. These include feature extraction using co-occurrence matrices [15, 30], feature extraction using Gabor filters [5, 6] and Markov random field modeling [1, 21, 23, 28, 36]. The second step is to apply a classifier on the feature set.

The approach elaborated in this paper uses Gabor filters $[3,4,5,6,9,10,20,24,25,28,29,31,36]$ for feature extraction, which is a slightly improved version of the algorithm proposed in [5]. The modified algorithm computes texture features using Gabor filters enhanced with local variance. The segmentation is achieved using k-means clustering followed by multi-class iterative Fisher Linear Discriminant $[5,6]$. The approach is rigorously tested on a wide varieties of several hundred textures with straight boundaries and varying sinusoidal boundaries from five texture databases viz. Outex texture database, Brodatz textures, Vistex textures, Prague textures and Pertex texture database [18, 22]. The image sizes used for testing are 256 by 256 and 512 by 512 . 
The texture segmentation using Gabor filters and Markov Random Field Modeling [1, 7, 21, 22, 23, 27, 28, 35, 36] by different researchers has been reviewed in the second section of the paper. Materials \& Methods section briefs the proposed algorithms / approach. Experimentation and result section depicts results of the proposed algorithm along with a comparison with ten state-of-the-art algorithms [22].

\section{Literature Review}

This section describes contributions of different researchers to texture segmentation and image segmentation approaches performed using Gabor filters and Markov random field (MRF) over the past twenty-eight years in a chronological order. This literature survey is divided into three categories. The first category focuses on the texture segmentation approaches using non-optimal Gabor filter parameters viz. center frequencies and orientations with reduced computational complexity $[9,10,20,24,25,29,31]$. The second category in the literature survey includes texture segmentation using optimal Gabor filter parameters and Fisher discriminant $[5,6]$ and segmentation of texture and sea ice synthetic aperture radar (SAR) images using a model-based approach based on MRF [7, 28, 34, 35]. An MRF-based approach is used to address the complex texture segmentation problem [28]. The third category includes the state-of-the-art segmentation approaches using MRF and those are reviewed towards the end of this section [22, 27].

Jain AK and Farrokhnia Farshid [20] proposed unsupervised texture segmentation using Gabor filters. The real component of Gabor filter outputs is used to reduce computations instead of magnitude of the filter output, without much improvement on relative benefits. This approach has a limitation that it requires an evaluation of the system as many times as the number of classes.

Dunn Dennis and Higgins WE [9, 10] proposed supervised texture segmentation of an image consisting of two textures separated by a straight boundary using Gabor filters. They recommended filters with minimum dominant frequencies to limit computational complexity. A single texture can have multiple dominant frequencies in it. Therefore, the texture segmentation approach proposed by them is not much effective practically.

Teuner Andreas et al [31] proposed unsupervised texture segmentation using Gabor filters. They estimated Gabor filter parameters using an analysis of spectral feature contrast matrix obtained using the evaluation of pyramidal Gabor transform. This approach eliminated the need of Gabor wavelet transform for unsupervised texture segmentation and at the same time reduced computational complexity and memory requirements.

Mao and Jain AK [24] proposed neural network-based supervised texture segmentation using Gabor filters. They reported the best results out of ten trials and reduced feature set to decrease computation time.

Panda R. and Chatterji BN [25] performed unsupervised texture segmentation using Gabor filters. They used K-means clustering algorithm, however they concluded that K-means clustering is not suitable for generic texture segmentation problem, since it works well only if clusters are hyper-spheroidal and these types of clusters are not possible in the case of all textures.

Randen T and Husoy JH [29] performed an exhaustive study of texture filtering approaches including Gabor filters developed in a decade from 1991 to 1999. They concluded that there was no single specific approach which offered optimum segmentation accuracy and computational complexity. They achieved a mean segmentation error of $34.3 \%$ on a few benchmark images of this decade using Gabor filters.

Clausi DA and Jernigan M Ed [6] presented an exhaustive study of supervised and unsupervised texture segmentation using different Gabor filter outputs. They studied six different feature extraction approaches based on different outputs of Gabor filter viz. magnitude of Gabor filter output, applying smoothing on Gabor filter outputs, only real component of outputs [20], rectification (absolute value of real and imaginary components) [2, 16]. Non-linear transformation of filter outputs [20], moments of Gabor filter outputs [3]. They proposed novel approaches namely local variance and local filter consistency of Gabor filter outputs to address a complex texture segmentation problem. It is inferred that the optimum texture segmentation is achieved with a magnitude response of Gabor filters and spatial frequency bandwidth of one octave with orientation spacing of $30^{\circ}$ and both filtering, smoothing performed in spatial frequency domain.

Clausi DA [5] suggested robust semi-supervised and unsupervised texture segmentation algorithms using Gabor filters. He noted in his publication that five iterations of Fisher linear discriminant were adequate for the segmentation of textures. The proposed separability measure for texture segmentation is Fisher distance, which is also termed as Fisher criterion. The algorithm was reported to be tested on seven diverse images.

Deng H and Clausi DA [7] proposed unsupervised sea-ice and texture segmentation using Gabor filters and Markov Random fields. They used Gabor filter for feature extraction. Feature energy was estimated assuming that feature data obeyed Gaussian distribution. The label energy was estimated using a second-order neighbourhood. They used a weighting factor between the feature and label energy components to determine contribution of individual component to the total amount of energy. The parameters viz. mean and variance were estimated using expectation maximization algorithm. The segmentation solution was obtained by minimizing the total amount of energy using Metropolis sampling and simulated annealing.

Yu Q and Clausi DA [34, 35] proposed an MRF based segmentation algorithm for highly non-stationary images. The traditional MRF based objective function consists of feature energy component and label energy component. The authors introduced an edge dependent penalty function in label energy component to address the segmentation of non-stationary images. The edge information is obtained using watershed segmentation algorithm. Optimization of global energy function is achieved by computing energy difference between the sum of the energy of the two merged segments minus the energy 
of each individual segment. They used two SAR sea-ice images for testing the performance of their algorithm and obtained a better qualitative result than the result obtained with a traditional MRF model and simulated annealing.

Qin AK and Clausi DA [28] extended an algorithm in [34, 35] for texture segmentation using Gabor filters and Markov random fields for highly complex texture segmentation. They achieved promising results on textures with high intra class variation. A segmentation solution space was reduced by performing initial over-segmentation using watershed transform followed by semantic region growing reducing computational complexity. The region adjacency graph was constructed on the over-segmentation and it was updated during optimization. Although they achieved good results, it was at a heavy computational cost and a complex theoretical MRF segmentation model.

Pereyra M and McLaughlin Steve [27] developed a computationally highly efficient unsupervised image segmentation algorithm using Potts Markov random field modeling. They estimated an MRF regularization parameter using a small variance asymptotic analysis of the improved Bayesian model and convex relaxation. This algorithm was tested on brain MRI, lungs and bacteria images and Synthetic Aperture Radar images. Their algorithm produced results as best as those produced by the state-of-the-art approaches.

Kiechle $\mathrm{M}$ et al [22] performed unsupervised texture segmentation using Mumford-Shah model on Prague texture database benchmark images $[14,22]$. The authors compared the performance of their algorithm with 10 state-of-the-art approaches. The filter set for estimating features of input images was obtained from patches of input texture images and from Prague texture segmentation database. They used 41 filters to learn features of texture images. The segmentation was achieved by solving piece-wise constant Mumford-Shah model. They applied this segmentation approach to Prague segmentation dataset and histological dataset. They achieved competitive results on both the datasets.

The literature survey clearly indicates that many researchers used Gabor filter for feature extraction with non-optimal parameters $[9,10,20,24,25,29,31]$. Some of the researchers used a computationally expensive and theoretically complex approach using MRF for the segmentation of textures and other images [7, 28, 34, 35]. Most of the researchers evaluated segmentation algorithm performance on a few texture images or a single texture image in some cases. An attempt has been made here to find a simple and computationally less expensive approach to improve texture segmentation accuracy. Gabor filters with optimum parameters are used for feature extraction in the proposed approach and this contributed to the increase in segmentation accuracy. A simple modification in a semi-supervised algorithm in [5] is suggested, which resulted into a significant increase in segmentation accuracy for highly complex textures and the combination of homogenous and complex textures with small texture primitives. The performance of the proposed algorithm is evaluated on several hundred images from five texture databases. These images include 90 benchmark images.

\section{Materials and Methods \\ Description of Semi Supervised Texture Segmentation Algorithm}

The texture segmentation algorithm needs feature data in a suitable form to discriminate texture segments using a classifier. The widely used feature extraction approach for textures is multichannel filtering performed using Gabor filters [4, 5, 6, 9, $10,20]$. The bank of Gabor filters, each with a center frequency and an orientation is used to generate a feature vector from the texture input image.

The texture segmentation algorithm presented here needs only the number of classes as an input. The algorithm provides computationally and theoretically simple approach for the segmentation of highly complex textures using Gabor filters [5, 6] and iterative multiclass Fisher discriminant compared to segmentation of textures performed using Markov Random Fields [7, 22, 28, 36].

The algorithm comprises the following steps.

1. Feature extraction of input texture image using Gabor filter with optimal parameters [6].

2. K-means clustering algorithm applied to a multi-dimensional feature set obtained in step- 1 .

3. Multiple iterations of Multi-class Fisher Linear discriminant (MFLD), used to refine segmentation results obtained in step-2 by using class parameters viz. mean and covariance obtained using K-means clustering.

Local variance of feature set is computed to enhance feature set, and then is deployed to improve segmentation results of complex textures [6].

As per reference [5], only 5-iterations of Fisher's discriminant are required to get good segmentation results. However, after exhaustive experimentation, it has been observed that typically 10-25 iterations are required for further improvement in segmentation results for complex textures. The improvement in texture segmentation accuracy is significant after increasing the number of iterations of MFLD. The Fisher distance is a measure of separability of textures in a multi-class input image. It increases as iterations of MFLD increase. It is further observed that Fisher distance increases beyond 5iterations for the case of highly complex textures or the combination of highly complex textures and homogeneous textures with small texture primitives, resulting into an increase of segmentation accuracy.

\section{Feature Extraction using Gabor Filter}

The input texture image is decomposed into 24 images and 30 images using a bank of Gabor filters for the image size of 256 by 256 and 512 by 512 respectively. Each filter has one center frequency and one orientation associated with it. Center frequencies used for the Gabor filters are $8 \sqrt{2}, 16 \sqrt{2}, \ldots,\left(\mathrm{N}_{\mathrm{c}} / 4\right) \sqrt{2}$ cycles/image width, where $\mathrm{N}_{\mathrm{c}}$ is the number of columns in the image $[6,20]$. The orientation spacing of $30^{\circ}$ and one octave bandwidth is used for each filter [6]. These are the optimal parameters for Gabor filter. The four center frequencies with six orientations form a feature vector of the size of 24. Appending spatial indices as texture features, forms a feature vector with a size of 26. 
The two-dimensional impulse response of Gabor filter is given in $[4,6]$

$$
h(x, y)=g\left(x^{\prime}, y^{\prime}\right) * \exp \left\{j 2 \pi F x^{\prime}\right\}
$$

where $\left(x^{\prime}, y^{\prime}\right)=(x \cos \emptyset+y \sin \emptyset,-x \sin \emptyset+y \cos \emptyset)$ are rotated co-ordinates and

$$
g(x, y)=\frac{1}{2 \pi \lambda \sigma_{x} \sigma_{y}} * \exp \left\{-\frac{1}{2}\left[\frac{(x / \lambda)^{2}}{\sigma_{x}^{2}}+\frac{y^{2}}{\sigma_{y}^{2}}\right]\right\}
$$

$\mathrm{F}$ is the center frequency and $\sigma_{\mathrm{x}}, \sigma_{\mathrm{y}}$ are standard deviations along $\mathrm{x}$-axis and y-axis respectively. As indicated in equation (1), the impulse response of Gabor filter is complex sinusoidal grating modulated by 2-D Gaussian function with an aspect ratio $\lambda$. The aspect ratio is defined as a ratio of the minor axis to major axis of Gaussian envelope in equation (2). The orientation of Gaussian envelope and sinusoidal grating is same for the impulse response as given in equation (1) and the same is denoted by $\phi$. Fig. 1a and Fig. 1b show intensity plots of the real and imaginary parts of impulse response of Gabor filter at six orientations viz. $0^{\circ}, 30^{\circ}, 60^{\circ}, 90^{\circ}, 120^{\circ}$ and $150^{\circ}$ for the center frequency of $\mathrm{F}=32$ cycles $/$ image and the aspect ratio of $\lambda=0.5$. The spatial frequency response of the Gabor filter is elliptical in shape as shown in Fig. 1c for $\lambda=0.5$. The horizontal lobe is associated with orientation $\phi=0^{\circ}$. The other elliptical lobes are associated with orientations of $30^{\circ}, 60^{\circ}$, $90^{\circ}, 120^{\circ}$ and $150^{\circ}$ in anticlockwise direction in Fig. 1c. The spatial frequency response is circularly symmetric for $\lambda=1$. The perspective views of real and imaginary parts of the impulse response of Gabor filter are shown in Fig. 1d and Fig. 1e respectively for the center frequency $\mathrm{F}=50$ cycles $/$ image and orientation $\phi=0^{0}$. Magnitude response of each Gabor filter is used as feature data. Each pixel in feature data image (decomposed image) is associated with one center frequency $\mathrm{F}$ and one orientation $\phi$.

The frequency domain representation of Gabor filter is -

$$
H(u, v)=\exp \left\{-2 \pi^{2}\left[(u-F)^{2} \sigma_{x}^{2}+v^{2} \sigma_{y}^{2}\right]\right\}
$$

The local variance of Gabor filter output is used as an enhanced feature to address the problem of the complex texture segmentation.

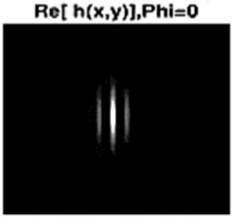

$\operatorname{lm}[h(x, y)], P h i=0$
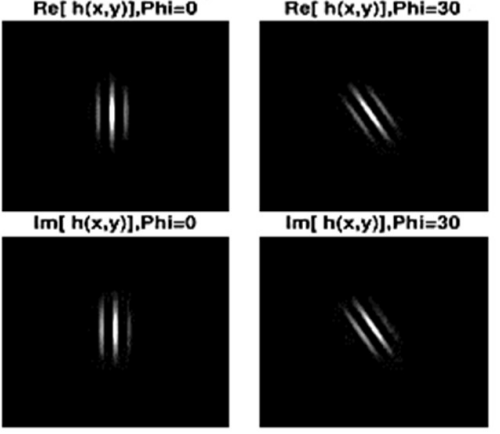

$\operatorname{lm}[h(x, y)], P h i=30$
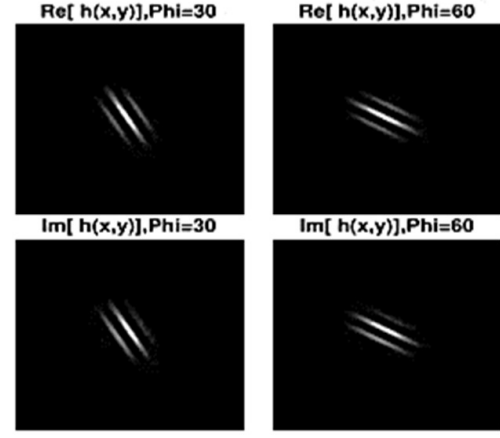

$\operatorname{Im}[h(x, y)], P h i=60$
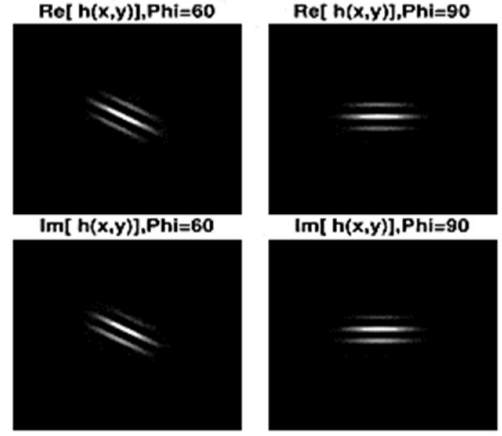

$\operatorname{Im}[\mathrm{h}(\mathrm{x}, \mathrm{y})], \mathrm{Ph}=90$
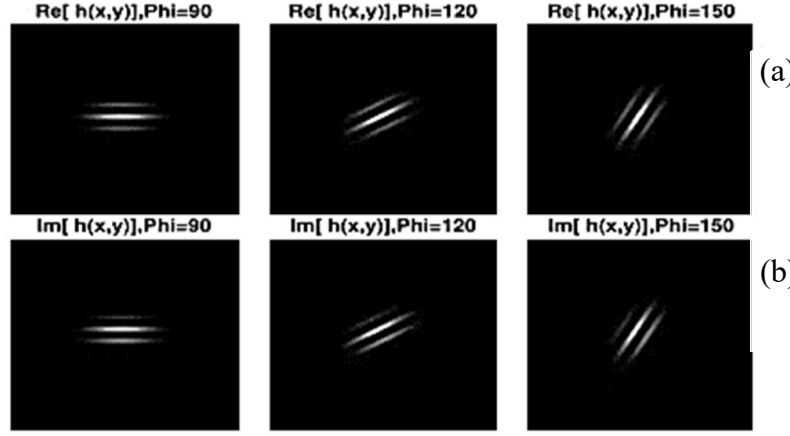

$\operatorname{Im}[h(x, y)], P h i=150$

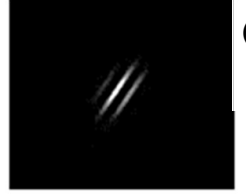

(b)

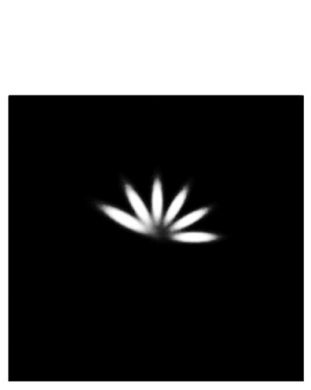

(c)

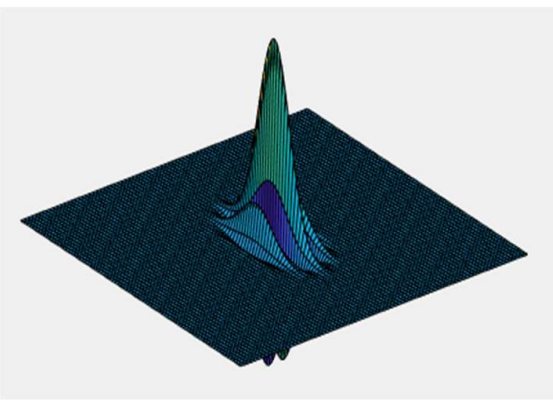

(d)

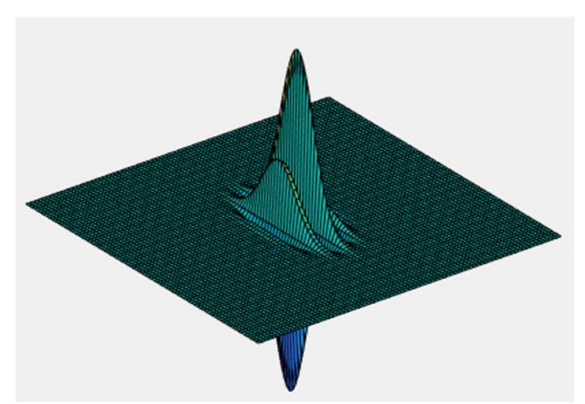

(e)

Fig. 1: (a) Real part of Impulse Response

(b) Imaginary Part of Impulse Response of Gabor Filter at Six Orientations at $0^{\circ}, 30^{\circ}, 60^{\circ}, 90^{\circ}, 120^{\circ}$ and $150^{\circ}$

(c) Spatial Frequency Response of Gabor Filter at the Same Six Orientations

(d) Perspective View of the Real Part of Gabor Filter for $F=50$ Cycles Per Image and Aspect Ratio $=0.5$

(e) Perspective View of Imaginary Part of Impulse Response of Gabor Filter for F $=50$ Cycles Per Image and Aspect Ratio $=0.5$

\section{K-means Clustering Algorithm}

$\mathrm{K}$-means clustering is recommended when texture data segments are hyper-spheroidal and Euclidean distance measure is used for clustering the data segments $[5,19]$. It is used as the first step after feature extraction in the proposed texture segmentation algorithm.

\section{Multi-class Fisher Linear Discriminant}


The iterative multi-class Fisher linear discriminant (MFLD) is used to increase the separation between two different classes and to reduce a spread within a class $[8,11]$. This results in an increase in the ratio of between-class scatter matrix $S_{\mathrm{B}}$ and within-class scatter matrix $S_{W}[26]$. This is achieved by a transformation of the feature data using an optimal projection vector. The optimal projection vector helps to separate textures in an image using Euclidean distance.

Let the total number of classes in the image be $\mathrm{C}$. In this case, the total (C-1) Eigen vectors need to be estimated. Let us denote feature data by X, projected feature data by $\mathrm{Y}$ and array of Eigen vectors by $\Theta$ as shown below.

$$
\begin{gathered}
X=\left\{x_{1}, x_{2}, \ldots \ldots \ldots, x_{m}\right\} \\
Y=\left\{y_{1}, y_{2}, \ldots \ldots \ldots, y_{c-1}\right\} \\
\Theta=\left\{\theta_{1}\left|\theta_{2}\right| \ldots \ldots \ldots \mid \theta_{c-1}\right\}
\end{gathered}
$$

Here $x_{1}, x_{2}, \ldots \ldots \ldots, x_{m}$ are vectors and $y_{1}, y_{2}, \ldots \ldots \ldots, y_{c-1}$ are scalars. The projected data for $\mathrm{i}^{\text {th }}$ Eigen vector $\theta_{\mathrm{i}}$ is given by $\mathrm{y}_{\mathrm{i}}$

$$
y_{i}=\theta_{i}^{T} X \text { or } Y_{i}=\Theta^{T} X
$$

The within class scatter matrix for multi-class FLD is given by $\mathrm{S}_{\mathrm{w}}$.

$$
\begin{gathered}
S_{W}=\sum_{i=1}^{C} S_{i} \\
\text { where } S_{i}=\sum_{i \in C_{i}}\left(x_{i}-u_{i}\right)\left(x_{i}-u_{i}\right)^{T} \\
u_{i}=\frac{1}{N_{i}} \sum_{i \in C_{i}}\left(x_{i}\right)
\end{gathered}
$$

The between class scatter matrix is given by

$$
S_{B}=\sum_{i=1}^{C} N_{i}\left(u_{i}-u\right)\left(u_{i}-u\right)^{T}
$$

$$
\text { where } u=\frac{1}{m} \sum_{i=1}^{m} x_{i}=\frac{1}{m} \sum_{i=1}^{m} N_{i} u_{i}
$$

Here, $u$ is the global mean of all texture segments and $u_{i}$ is the mean of $i^{\text {th }}$ texture segment. The feature data is projected on the array of Eigen vectors $\Theta$.

$$
\begin{aligned}
& \hat{u}_{i}=\text { Mean of projected } \mathrm{i}^{\text {th }} \text { segment }=\frac{1}{N_{i}} \sum_{i \in C_{i}} y_{i}=\hat{u}_{i} \\
& \text { Global mean }=\frac{1}{N} \sum_{i=1}^{m} y_{i}=\hat{u}_{i}
\end{aligned}
$$

The expression for within-class scatter matrix and between class scatter matrix in the projected space are given in the following expressions.

$$
\begin{aligned}
& \hat{S}_{W}=\sum_{i=1}^{C} \sum_{i \in C_{i}}\left(y_{i}-\hat{u}_{i}\right)\left(y_{i}-\hat{u}_{i}\right)^{T} \\
& \hat{S}_{B}=\sum_{i=1}^{C} N_{i}\left(\hat{u}_{i}-\hat{u}\right)\left(\hat{u}_{i}-\hat{u}\right)^{T}
\end{aligned}
$$

The relation between the scatter matrices and array of Eigen vectors can easily be proved and it is as given below.

$$
\hat{S}_{W}=\Theta^{T} S_{W} \Theta \text { and } \hat{S}_{B}=\Theta^{T} S_{B} \Theta
$$

The Fisher criterion (Fisher distance) for multi-class FLD is given by the following expression.

$$
J(\Theta)=\frac{\left|\hat{S}_{B}\right|}{\left|\hat{S}_{W}\right|}=\frac{\left|\Theta^{T} S_{B} \Theta\right|}{\left|\Theta^{T} S_{W} \Theta\right|}
$$

The optimal projection vectors $\Theta^{*}$ is obtained by solving the generalized Eigen value problem.

$$
\begin{aligned}
& \left(S_{B}-\lambda_{i} S_{W}\right) \theta_{i}^{*}=0 \\
& \Theta^{*}=\left[\theta_{1}^{*}\left|\theta_{2}^{*}\right| \ldots \ldots \ldots \mid \theta_{c-1}^{*}\right]
\end{aligned}
$$

The segmentation of a multi-texture image is achieved by projecting the image on the array of the optimal projection vector $\Theta^{*}$ and by applying MFLD classifier to it.

\section{Implementation of Iterative MFLD}

The crude segmentation results and centroids of the texture segments in the input texture image are obtained using K-means clustering from the enhanced feature sets. These rough segmentation results are refined using iterative MFLD as described below.

Step 1: Compute within-class scatter matrix $S_{w}$ and between-class scatter matrix $S_{B}$ from feature data $x_{i}$ and centroids $u_{k}$ estimated by k-means clustering using eq. (5) and eq. (6). Here, k associated with centroids is assumed on the values of $1,2, \ldots \ldots, \mathrm{C}$, where $\mathrm{C}$ is the total number of texture segments in the input image.

Step 2: Estimate projection vector $\Theta^{*}$ with an improved direction by solving eq. (12) using within-class scatter matrix $S_{\mathrm{w}}$ and between-class scatter matrix $\mathrm{S}_{\mathrm{B}}$.

Step 3: Project feature data $x_{i}$ and centroids $u_{k}$, on the projection vector $\Theta^{*}$ obtained in Step 2.

Step 4: Refine segmentation (labeling) using Euclidean distance from the projected data $\mathrm{y}_{\mathrm{i}}$ in eq. (4).

Step 5: Compute centroids $u_{k}$, within-class scatter matrix $S_{w}$ and between-class scatter matrix $S_{B}$ from feature data associated with refined labels in step 4 using eq. (5) and eq. (6). 
Step 6: Stop iterations if average of Fisher distance $\mathrm{J}(\Theta)$ i.e. ratio of determinant $\left|\Theta^{T} \boldsymbol{S}_{\boldsymbol{B}} \Theta\right|$ to determinant $\left|\Theta^{T} \boldsymbol{S}_{\boldsymbol{W}} \Theta\right|$ in eq. (11) ceases to increase or go to step 2.

\section{Experimentation and Results}

The proposed algorithm is exhaustively tested on five databases $[14,18]$ viz. Outex texture database developed by Oulu University, Finland, Europe, Vision Texture database developed by MIT, Boston, USA, Brodatz texture database, Prague texture segmentation database and Pertex texture database. The performance of the proposed algorithm is compared with ten state-of-the-art algorithms. All of these algorithms are evaluated on Prague texture database benchmark images. The state-of-the-art algorithms include the one proposed by Kiechle M et al [22]. The other nine algorithms are Priority MultiClass Flooding Algorithm (PMCFA), Variational Multi-Phase Segmentation (PCA-MS), Factorization based Segmentation (FSEG), Regression based Segmentation RS, Texture Fragmentation and Reconstruction (TFR), 3D Auto Regressive Model (AR3D), Gaussian Markov Random Field with Expectation Maximization GMRF+EM, Texture Segmentation by Weighted Aggregation (SWA) and Texel-Based Segmentation (TS) [22]. Algorithm TS is evaluated on ten benchmark images, AR3D, GMRF+EM, SWA are evaluated on 20 images and the remaining algorithms are evaluated on 80 images.
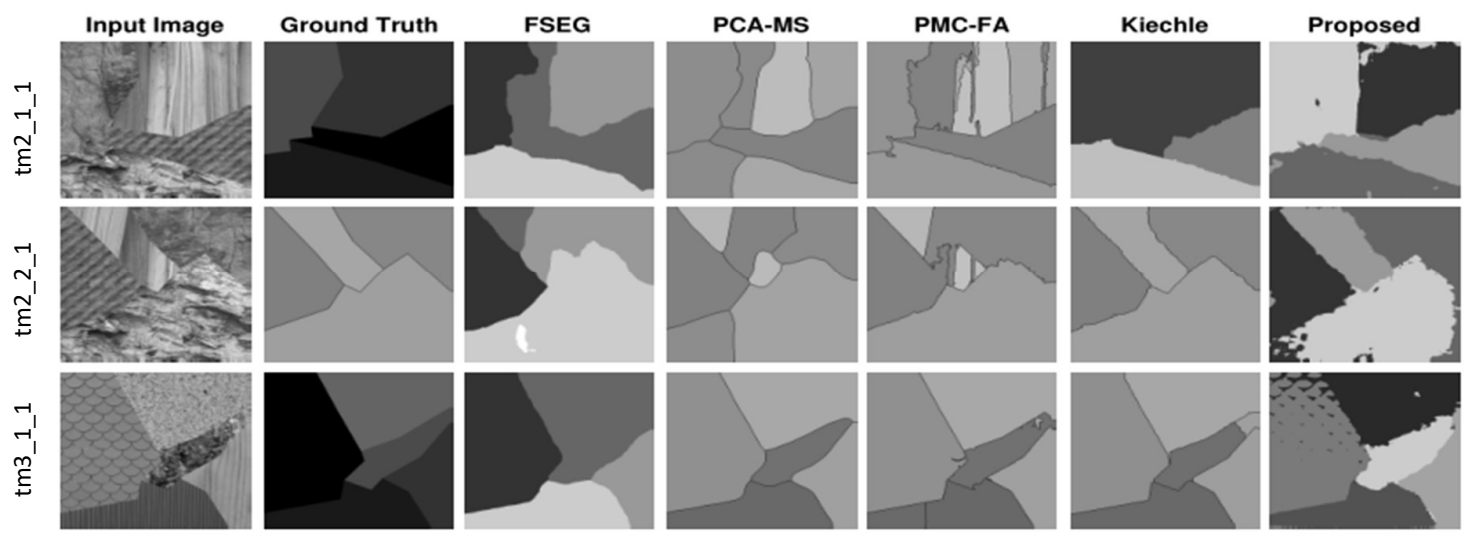

(a)
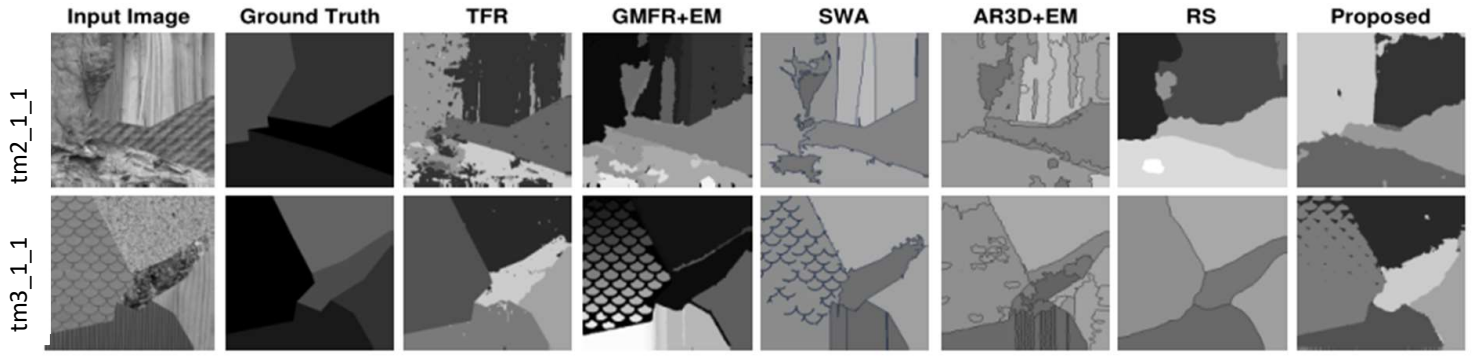

(b)

Fig. 2: (a) and (b) Comparison of the Performance of the Proposed Algorithm with Nine ${ }^{\mathrm{e}}$ State of the Art Approaches on Three Prague Texture Segmentation Benchmark Images

Note: ${ }^{\mathrm{e}}$ Images for Texel Based Segmentation Results were not available and therefore not shown in above diagram.

The Prague texture segmentation benchmark image set consists of 80 multi-class textures. These images are developed from 114 textures in Prague texture database images of 10 different categories [22]. The size of all the images is 512 by 512. This database consists of grayscale and color homogeneous textures and complex textures with both large and small texture primitives relative to the size of the image. The proposed algorithm in this study is tested on grayscale of 130 $(80+50)$ multi-class images with different boundaries. It is observed after exhaustive experimentation that Fisher distance which is a measure of texture separability increases by a significant amount for images containing complex texture with very small texture primitives relative to the size of the image. Fig. 2a and Fig. $2 b$ show a segmentation performance of the proposed algorithm and the state-of-the-art algorithms in [22] on some Prague benchmark images. It is clear that the proposed algorithm achieves better results than most of the state-of-the-art algorithms. The proposed method achieves the second rank on 21 benchmark images among the ten state-of-the-art approaches as discussed in result analysis and discussion section of this paper. Fig. 3a shows an iteration-wise improvement in the segmentation performance of 4-class image from Prague texture database using optimal Gabor filter parameters as stated previously in section on feature extraction using Gabor filter. The segmentation accuracy at the $5^{\text {th }}$ iteration is $74.14 \%$ and the accuracy at the $22^{\text {nd }}$ iteration is $95.88 \%$ yielding an accuracy gain of $21.74 \%$. Fig. $3 \mathrm{~b}$ shows a graph of segmentation accuracy against Fisher distance and it indicates that the accuracy increases with an increase in Fisher distance. A detailed justification is provided for the images in Prague benchmark set for which segmentation results are not good in result analysis and discussion section of this paper. 


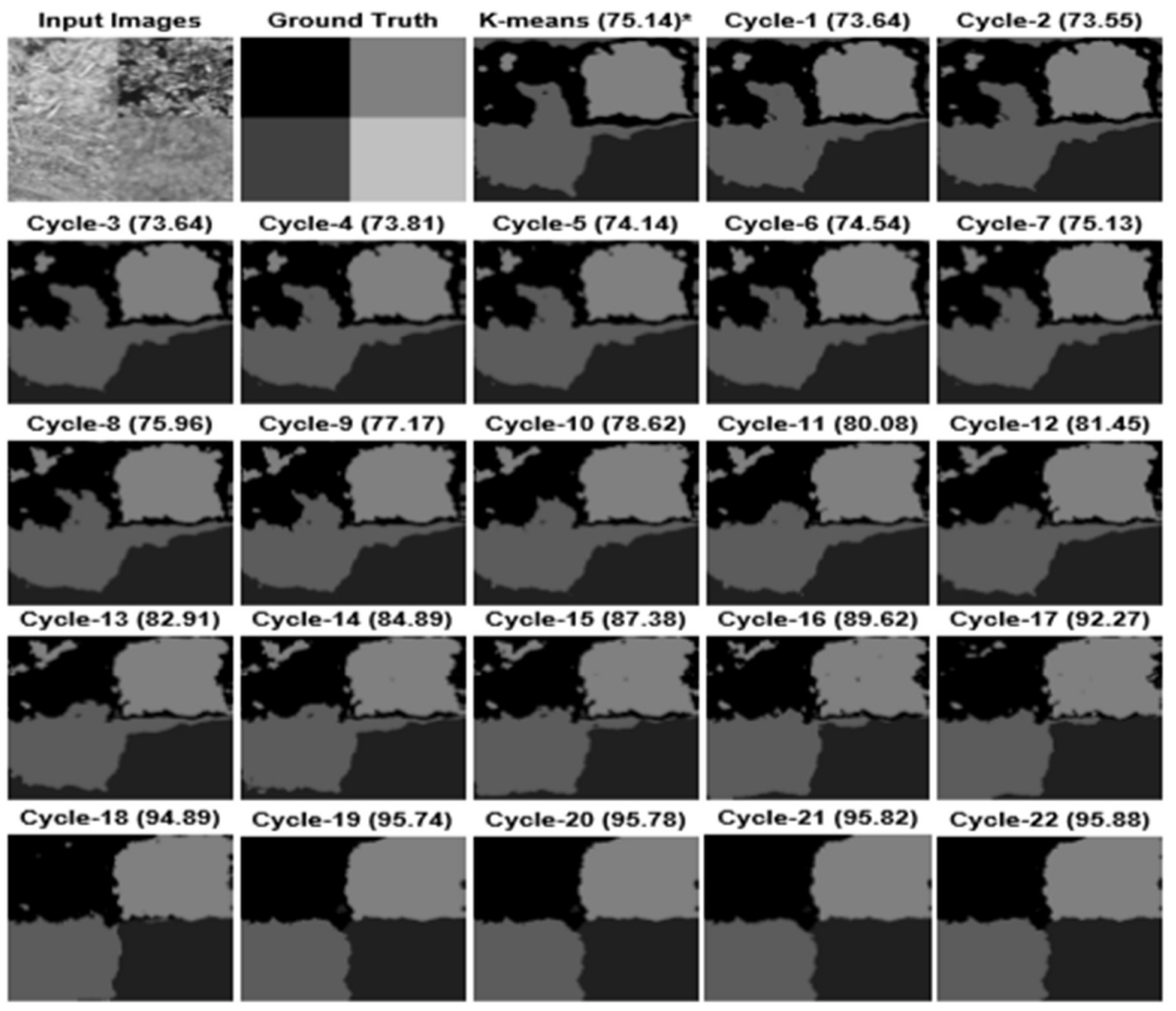

Fig. 3a: Iteration-Wise Results of Four Class Prague Texture Database Image with Optimal Gabor Filter Parameter Set ${ }^{\mathrm{f}}$

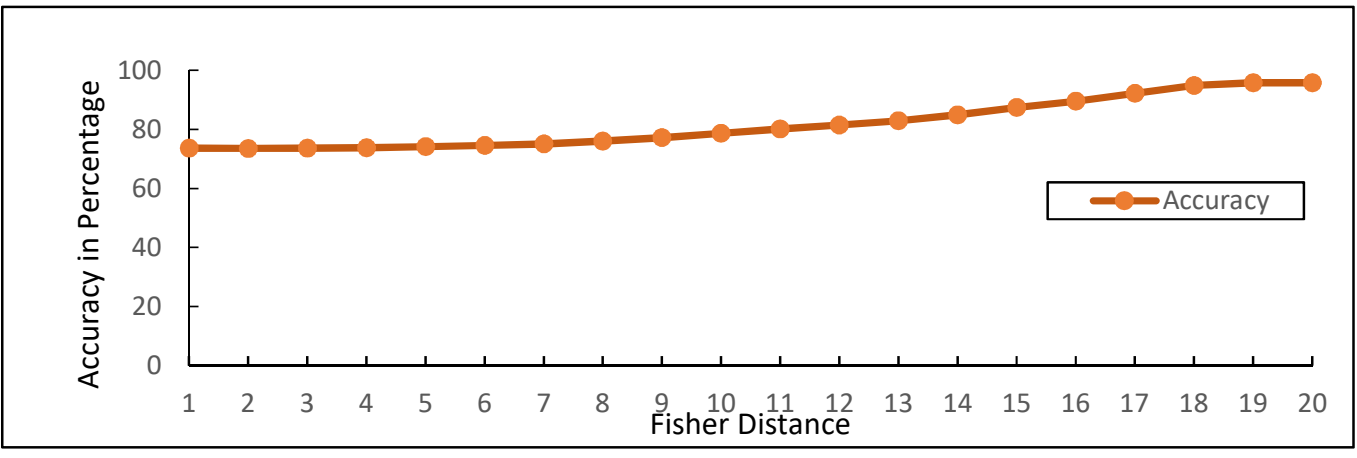

Fig. 3b: A Graph of Segmentation Accuracy vs Fisher Distance

Note: *Values in round brackets in the above Fig. 3a indicates segmentation accuracy for the given cycle.

Note: ${ }^{f}$ The input image contains 4 textures from Prague texture database viz. droppings, hedge, kaolin5 and kaolin 1

Brodatz texture database has 112 images with a size of 640x640 [18]. These textures are pictures of an album developed for artistic intent. These textures are of good quality because they are captured under controlled lighting conditions. However, they have poor intra-class variation. This database contains some textures with good diversity, some with similarity, but some textures are inhomogeneous [18]. The textures with straight boundaries and varying sinusoidal boundaries in the terms of different amplitudes and different cycles, over the edges of the boundaries, are used for testing the performance of the proposed algorithm. The algorithm is tested on $127(50+25+41+11)$ combinations of $512 \times 512$ size textures with five segments and different sinusoidal boundaries. The segmentation accuracy increases with an increase in the number of iterations (cycles) of MFLD. Complex fine textures are separated with an increasing number of cycles of 
MFLD. Fig. 4a shows the iteration-wise improvement in segmentation accuracy for 4-segments Brodatz texture image T8 for 27 cycles with optimal Gabor filter parameter set. The segmentation accuracy is increased by $23.36 \%$ in $27^{\text {th }}$ cycle relative to $5^{\text {th }}$ cycle. Fig. $4 \mathrm{~b}$ shows a graph of segmentation accuracy against Fisher distance for 4-segment Brodatz image T8 with straight boundaries and it indicates that accuracy increases with an increase in FD.
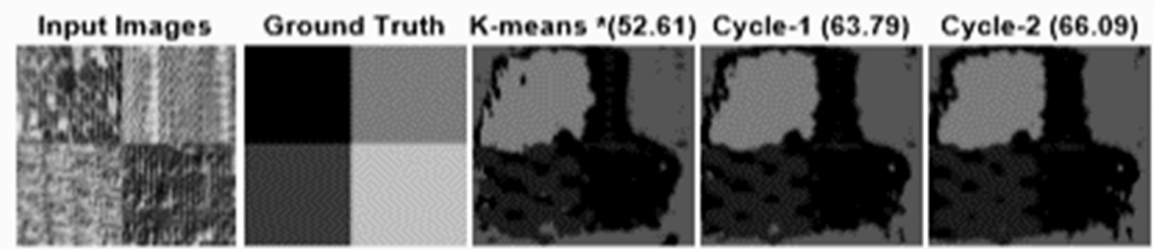

Cycle-3 (68.28)

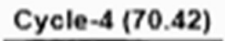

Cycle-5 (72.39)

Cycle-6 (73.97)

Cycle-7 (75.41)

Cycle-8 (76.82)
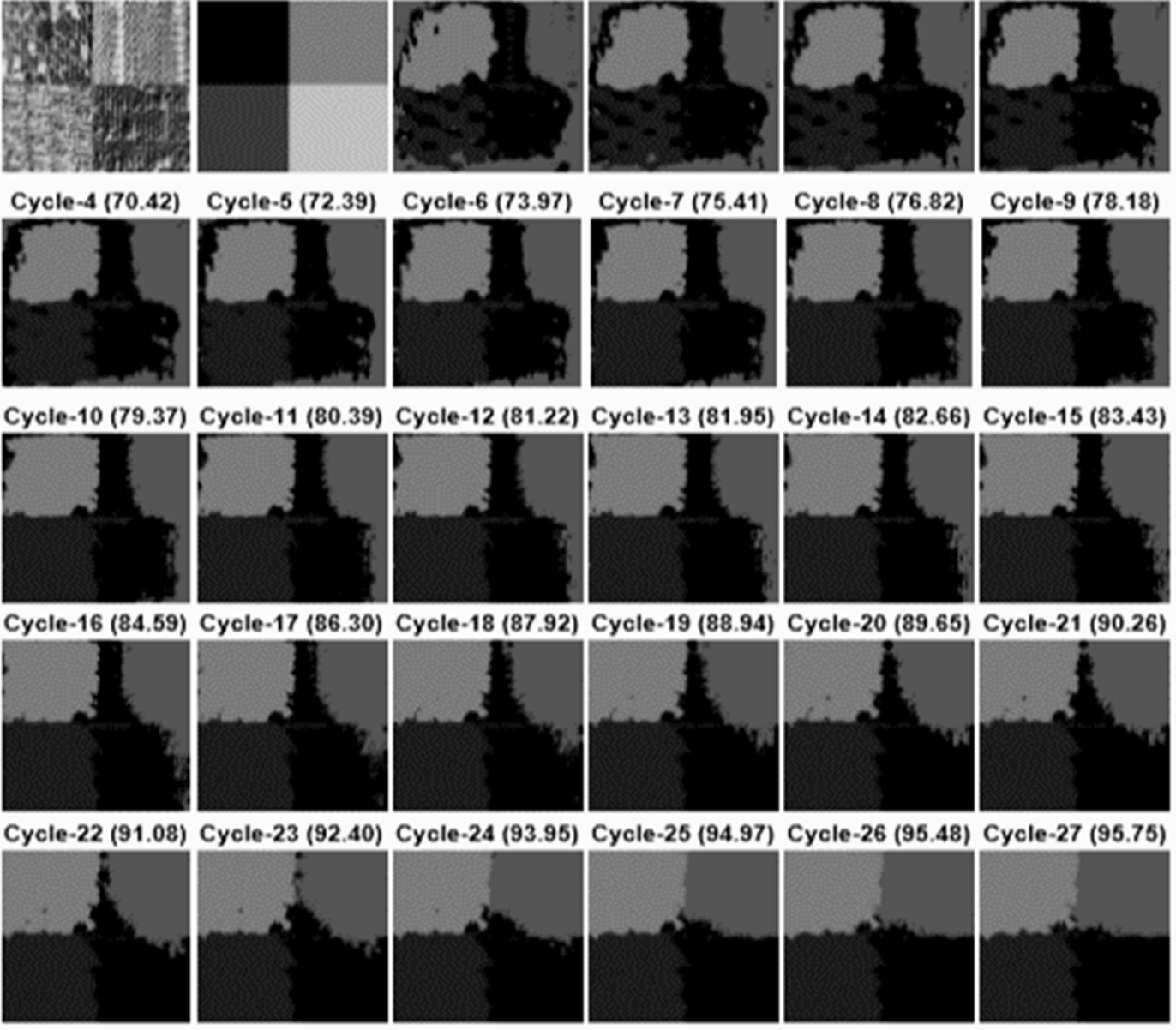

Fig. 4a: Iteration-Wise Results of Four Class Brodatz Texture Database Image with Optimal Gabor Filter Parameter Set ${ }^{\mathrm{g}}$

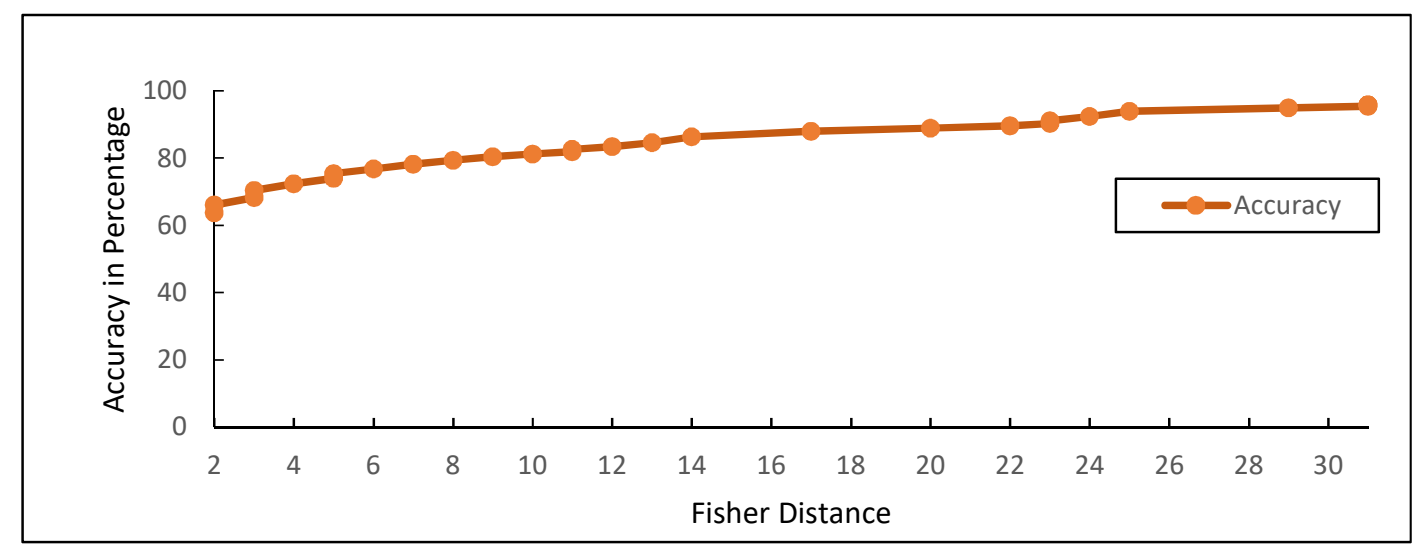

Fig. 4b: A Graph of Segmentation Accuracy vs Fisher Distance

Note: * Values in round brackets in the above Fig.4a indicates segmentation accuracy for the given cycle. Note: ${ }^{\mathbf{g}}$ The input image contains 4 textures from Brodatz texture database viz. D81, D85, D82 and D80 
Fig.5a:shows results for Brodatz texture image, with five texture segments with varying sinusoidal boundaries, with optimal Gabor filter parameter set and it indicates that the segmentation accuracy is increased by $7.95 \%$ for five texture images at the cycle number 20 relative to the cycle number 5 . Fig. 5b shows a graph of accuracy against FD and it indicates that the accuracy increases with an increase in FD. The algorithm is tested on more than 100 4-segment textures and 100 2-segment textures with the size of 256x256. It is further tested on 25 textures with 5- segment texture images with circular and straight boundaries.
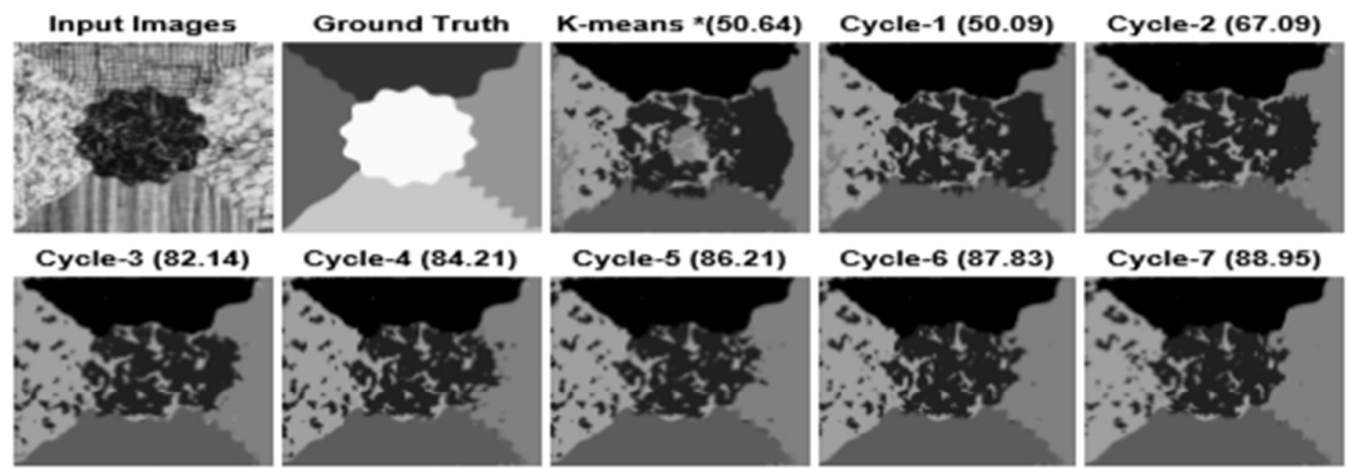

Cycle-6 (87.83)
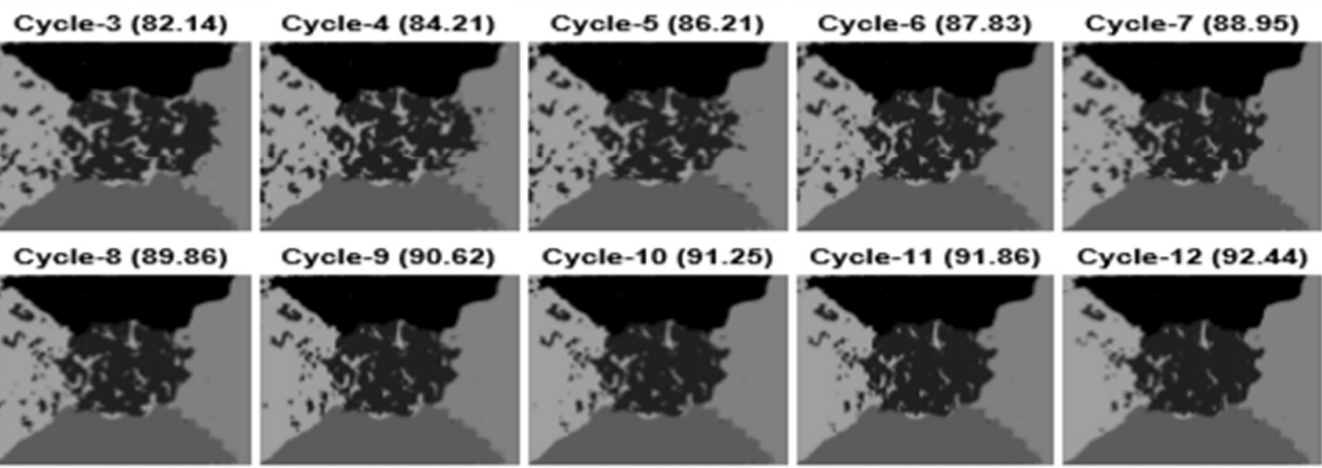

Cycle-9 (90.62)
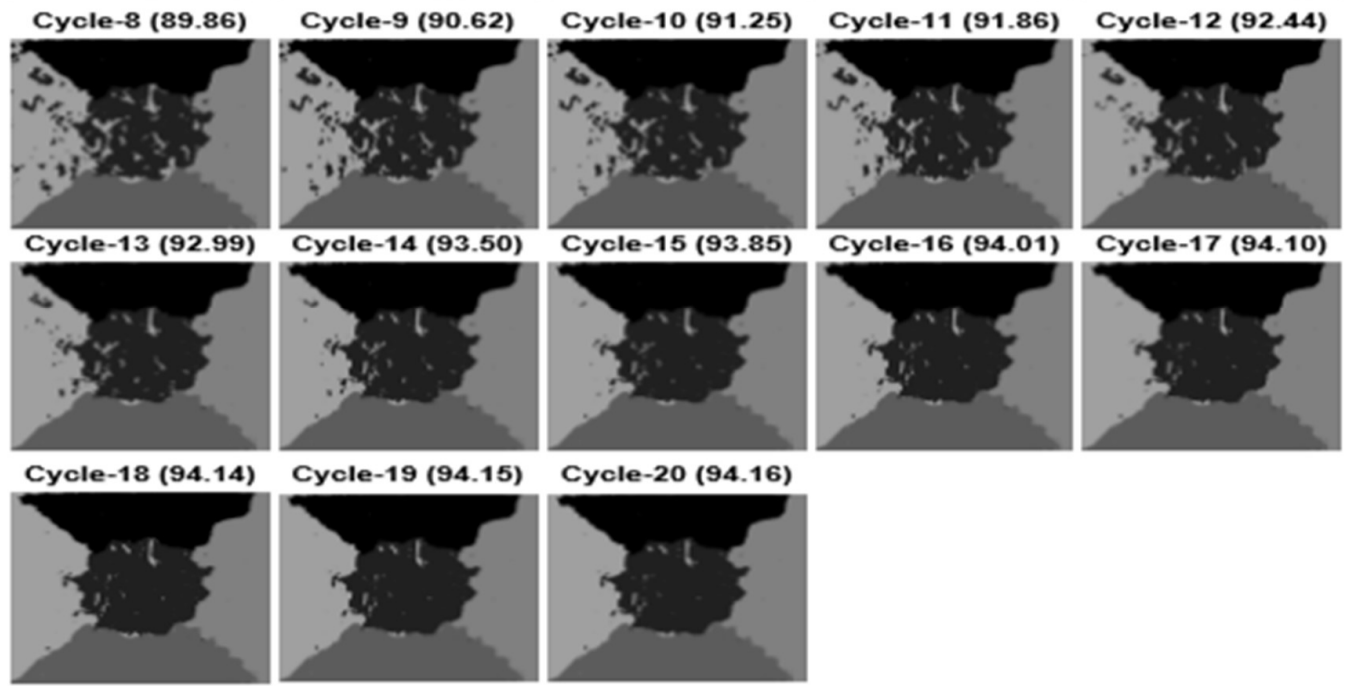

Cycle-20 (94.16)

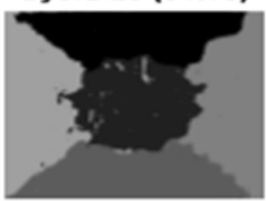

Fig.5a: Iteration-Wise Results of Five Class Brodatz Texture Database Image with Optimal Gabor Filter Parameter Set ${ }^{\text {h }}$

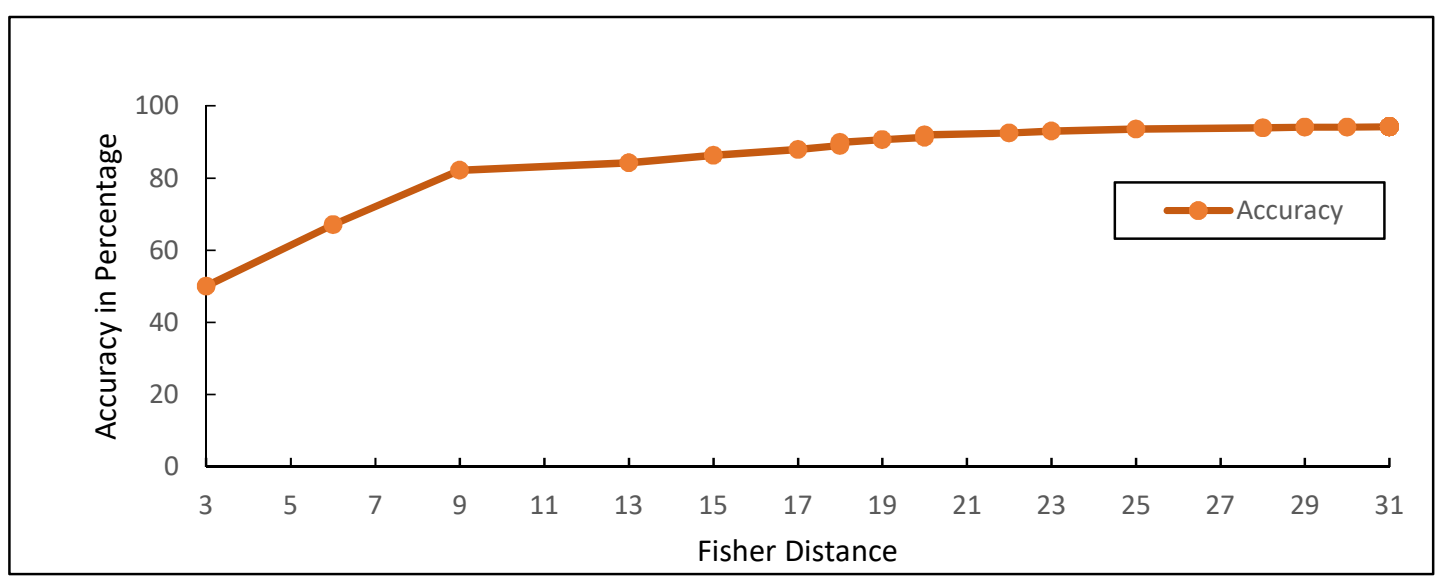

Fig. 5b: A Graph of Segmentation Accuracy vs Fisher Distance

Note: *Values in round brackets in the above Fig. 5a indicates segmentation accuracy for the given cycle.

Note: ${ }^{\mathbf{h}}$ The input image contains 5 textures from Brodatz texture database viz. D110, D112, D104, D106 and D109 
Table 1 shows that segmentation accuracy increases as iterations of MFLD increase for Brodatz texture database.

Outex texture database contains surface textures and natural scenes. There are a total of 320 textures in this database. The surface textures have variations in the form of illumination, rotation and spatial resolution. Each texture is captured with six different spatial resolutions viz. 100, 200, 300, 360, 500 and $600 \mathrm{dpi}$ and nine rotation angles viz. $0^{0}, 5^{\circ}, 10^{\circ}, 15^{\circ}, 30^{\circ}$, $45^{\circ}, 60^{\circ}, 75^{\circ}$ and $90^{\circ}$.

Three test suites are provided in this database conducive for, namely, supervised texture segmentation (SS), unsupervised texture segmentation (US) and texture classification (TC). The proposed algorithm is tested on US texture data suite having hundred 512x512 images therein with varying sinusoidal boundaries. 66 images out of 100 have a segmentation accuracy above $83 \%$, nine textures have an accuracy above $95 \%$, twelve textures have an accuracy above $94 \%$ and below $95 \%, 13$ images have an accuracy above $93 \%$ and below $94 \%$ etc. The boundaries are retained well after segmentation for the combination of homogeneous and complex textures. This justifies the robustness of algorithm. Table 2 shows that the segmentation accuracy increases as iterations of MFLD increase for Outex texture database.

\begin{tabular}{|c|c|c|c|c|c|c|c|}
\hline $\begin{array}{c}\text { Input Image } \\
\text { No. }{ }^{i} \\
\end{array}$ & $\begin{array}{l}\text { Accuracy } \\
5 \text { Cycles } \\
\end{array}$ & $\begin{array}{l}\text { Accuracy } \\
10 \text { Cycles }\end{array}$ & $\begin{array}{c}\text { Improvement }^{\mathbf{k}} \\
\text { in Accuracy }\end{array}$ & $\begin{array}{l}\text { Accuracy } \\
15 \text { Cycles }\end{array}$ & $\begin{array}{c}\text { Improvement } \\
\text { in Accuracy }\end{array}$ & $\begin{array}{l}\text { Accuracy } \\
30 \text { Cycles }\end{array}$ & $\begin{array}{c}\text { Improvement } \\
\text { in Accuracy }\end{array}$ \\
\hline 1. & 70.0371 & 71.2334 & 1.1963 & 86.813 & 16.7759 & 86.9537 & 16.9166 \\
\hline 2. & 89.8094 & 90.8779 & 1.0685 & 91.6893 & 1.8799 & 93.7931 & 3.9837 \\
\hline 3. & 93.6665 & 94.2169 & 0.5504 & 94.2978 & 0.6313 & 94.3016 & 0.6351 \\
\hline 4. & 68.0027 & 95.1435 & 27.1408 & 95.285 & 27.2823 & 95.4311 & 27.4284 \\
\hline 5. & 64.217 & 65.1382 & 0.9212 & 69.3047 & 5.0877 & 94.0521 & 29.8351 \\
\hline 6. & 86.2129 & 91.2571 & 5.0442 & 93.853 & 7.6401 & 94.1517 & 7.9388 \\
\hline 7. & 66.9399 & 95.2399 & 28.294 & 95.6413 & 28.7014 & 95.7085 & 28.7686 \\
\hline 8. & 89.8308 & 91.2773 & 1.4465 & 92.8211 & 2.9903 & 93.7138 & 3.883 \\
\hline 9. & 90.0261 & 90.8958 & 0.8697 & 91.6325 & 1.6064 & 92.4934 & 2.4673 \\
\hline
\end{tabular}

$\begin{array}{ll}\text { Note: }^{\mathrm{i}} & \text { 1. D2-D3-D5-D1-D9 } \\ & \text { 4. D55-D52-D50-D51-D56 } \\ & \text { 7. D76-D29-D24-D9-D77 }\end{array}$

Table 1: Brodatz Texture Database Results
2. D20-D28-D38-D36-D22
3. D49-D50-D51-D52-D36
5. D92-D84-D76-D80-D95
6. D110-D112-D104-D106-D109
8. D29-D37-D63-D80-D105
9. D2-D4-D5-D9-D19

\begin{tabular}{|c|c|c|c|c|c|c|c|}
\hline $\begin{array}{l}\text { Sr. } \\
\text { No. }\end{array}$ & $\begin{array}{c}\text { Input } \\
\text { Image }\end{array}$ & $\begin{array}{l}\text { Accuracy } \\
5 \text { Cycles }\end{array}$ & $\begin{array}{l}\text { Accuracy } \\
10 \text { Cycles }\end{array}$ & $\begin{array}{c}\text { Improvement }^{\mathrm{k}} \\
\text { in Accuracy }\end{array}$ & $\begin{array}{l}\text { Accuracy } \\
15 \text { Cycles }\end{array}$ & $\begin{array}{c}\text { Improvement } \\
\text { in Accuracy }\end{array}$ & $\begin{array}{l}\text { Accuracy } \\
30 \text { Cycles }\end{array}$ \\
\hline 1. & problem-21 & 82.3456 & 94.9764 & 12.6308 & 94.994 & 12.6484 & 94.9966 \\
\hline 2. & problem-40 & 88.6982 & 94.3981 & 5.6999 & 94.8238 & 6.1256 & 94.8486 \\
\hline 3. & problem-50 & 90.0646 & 93.792 & 2.8274 & 94.1292 & 4.0646 & 94.3031 \\
\hline 4. & problem- 60 & 92.3679 & 94.3943 & 2.0264 & 94.4958 & 2.1279 & 94.5045 \\
\hline 5. & problem-61 & 93.7149 & 94.8296 & 1.1147 & 95.3938 & 1.9466 & 95.6615 \\
\hline 6. & problem-69 & 68.2087 & 86.7142 & 18.5055 & 90.995 & 22.7863 & 94.915 \\
\hline 7. & problem-98 & 89.8453 & 93.2438 & 3.3985 & 95.1324 & 5.2871 & 95.1065 \\
\hline \multicolumn{8}{|c|}{ Table 2: Outex Texture Database Results } \\
\hline $\begin{array}{c}\text { Input } \\
\text { Image } \\
\text { No. }^{\mathrm{m}}\end{array}$ & $\begin{array}{r}\text { Accuracy } \\
5 \text { Cycles }^{j}\end{array}$ & $\begin{array}{l}\text { Accuracy } \\
10 \text { Cycles }\end{array}$ & $\begin{array}{l}\text { Improvement }^{k} \\
\text { in Accuracy }\end{array}$ & $\begin{array}{l}\text { Accuracy } \\
15 \text { Cycles }\end{array}$ & $\begin{array}{l}\text { Improvement } \\
\text { in Accuracy }\end{array}$ & $\begin{array}{l}\text { Accuracy } \\
30 \text { Cycles }\end{array}$ & $\begin{array}{l}\text { Improvement } \\
\text { in Accuracy }\end{array}$ \\
\hline 1. & 80.7217 & 90.3809 & 9.6592 & 90.4739 & 9.7522 & 90.4934 & 9.7717 \\
\hline 2. & 81.2092 & 86.0718 & 4.8626 & 88.5582 & 7.349 & 88.979 & 7.7698 \\
\hline 3. & 64.917 & 65.4816 & 0.5646 & 75.1968 & 9.752 & -- & No \\
\hline 4. & 84.8927 & 90.2683 & 5.3756 & 92.5232 & 7.6305 & 92.7998 & 7.9071 \\
\hline 5. & 90.4766 & 91.819 & 1.3424 & 92.1459 & 1.6693 & -- & No \\
\hline
\end{tabular}

Table 3: VisTex Texture Database Results

Note: ${ }^{m}$ 1. Misc3-vis4-Misc2-vis4 -Metal5-vis4-M4-vis4-M3-vis4 2. Sand5-vis5-St5-vis5-M5-vis4-Misc3-vis4-Tile0-vis5

3. Metal2-vis4-M3-vis4-M4-vis4-Misc3-vis4-Misc2-vis4 4. Fabric19-vis3-F18-vis3-F17-vis3-F7-vis3-Metal0-vis4

5. Metal1-vis4-M2-vis4-M5-vis4-Misc2-vis4-S2-vis5

Note: i,l,m All input images in all the tables are 512x512 in size with varying sinusoidal boundaries. The title of each table indicates the name of texture database. The number in the first column of Table 4 and Table 6 indicate the name of textures in the corresponding database.

Note: ${ }^{\mathrm{j}}$ Cycles in each table indicates iterations of MFLD for the relevant texture database name in the title of table. Note: ${ }^{\mathbf{k}}$ Improvement in accuracy column in each table indicates improvement in accuracy relative to 5 iterations of MFLD. 
Vision Texture Database (Vistex) is developed by MIT Media Lab, Boston, USA. It covers extensive varieties of traditional and non-traditional real-world textures captured under natural lighting conditions. These textures demand for highly robust texture segmentation algorithm. The proposed algorithm is tested on more than 50 Vistex textures with a size of $256 x 256$. Some of the textures are combinations of homogeneous and complex textures and some are highly complex textures. The segmentation accuracies higher than $94 \%$ and as high as $98.52 \%$ are achieved for Vistex- 6 and Vistex-3 textures in this database. Table 3 indicates that the segmentation accuracy improves as iterations of MFLD increase for different cycles. The size of each input image in all the tables (Table 1, Table 2 and Table 3) are 512 by 512 with varying sinusoidal boundaries. The cycles in these tables indicate iterations of MFLD. The improvement in accuracy for different cycles is relative to $5^{\text {th }}$ cycle of MFLD.

Pertex texture database is developed by Texture lab Edinburgh, United Kingdom. It has a total of 334 textures in it. Some of the textures are complex and some are homogenous. The proposed algorithm is tested on 50 homogenous textures having the size of $512 \times 512$. The segmentation accuracies for some of the textures are $94 \%$ and some have accuracies as high as $97.47 \%$. The proposed algorithm is not tested on complex textures for this database.

\section{Result Analysis and Discussion}

Table 4 shows a comparison of the performance of the proposed algorithm based on highly exhaustive experimentation with ten state-of-the-art algorithms based on Hoover's segmentation metric 'correct segmentation' (CS) as discussed in [17, 22] for Prague benchmark images. This metric compute correctly labeled percentage of pixel as accuracy by comparing ground truth with segmentation results [17].

\begin{tabular}{|c|c|c|c|c|c|c|c|c|c|c|c|}
\hline Metric & Proposed & Kiechle & PMCFA & PCA-MS & FSEG & RS & TFR & AR3D & GMRF & SWA & TS \\
\hline CS & 75.35 & 77.73 & 75.32 & 72.27 & 69.02 & 46.02 & 46.13 & 37.24 & 31.93 & 27.04 & 59.1 \\
\hline
\end{tabular}

Table 4: Results Comparison with Ten State-of-the-Art Algorithms

Each value in Table 4 is a mean of the segmentation accuracies computed for Prague database benchmark images for specified algorithms based on CS segmentation metric [17]. This method achieves the second rank in the performance for the images in Prague texture segmentation benchmark on 21 images as indicated in Table 4. The benchmark images for which the proposed algorithm does not perform well, a detailed justification is provided in this section.

The algorithm proposed in this paper is based on optimal configuration of Gabor filter for texture segmentation presented in $[6,9]$ for feature extraction. Exhaustive testing with the proposed algorithm is performed using optimal parameter values for Gabor filter. The optimal parameters of Gabor filter are stated previously in section on feature extraction using Gabor filter. Local variance of Gabor filter outputs is computed to address complex texture segmentation problem. This feature extraction step is optimum and contributed to achieve the second rank in the ten state-of-the-art algorithms. Apart from this, Gabor filter is developed based on the human visual system. There are some textures in benchmark images whose boundaries are not easily discriminated even by a human eye. Some texture boundaries are difficult to discriminate due to a very gradual change in pixel values across the boundaries [12], very similar neighbouring textures, same frequencies and orientation in texture images resulting into a degradation of the image segmentation performance.

The second step in the algorithm is MFLD classifier. The classifier performance is decided by the value of the Fisher distance given in eq. (11). If it increases, the direction of the projection vector improves resulting into an increase in segmentation accuracy. The increase in the accuracy is significant only if Fisher distance increases significantly over the iterations. The extensive experimentation indicates that such situation occurs for highly complex textures or the combination of homogeneous and complex textures with small texture primitives relative to the size of the image (fine complex textures). The texture primitives in many benchmark images of Prague texture data set are large which results into the degradation of segmentation performance. If the transition across the texture boundaries is not abrupt, the classifier performance degrades. The gray level across boundaries for many texture images in Prague benchmark set is changing gradually degrading segmentation results.

\begin{tabular}{|c|c|c|c|c|}
\hline $\begin{array}{l}\text { Sr. } \\
\text { No. }\end{array}$ & $\begin{array}{c}\text { Image } \\
\text { name }\end{array}$ & $\begin{array}{c}\text { Accuracy with MIRGS } \\
\text { algorithm [28] }\end{array}$ & $\begin{array}{l}\text { Accuracy with the proposed } \\
\text { modification for } 5^{\text {th }} \text { iteration }\end{array}$ & $\begin{array}{c}\text { Accuracy with the } \\
\text { proposed modification }\end{array}$ \\
\hline 1. & T2 [28] & $97.3 \%$ & $94.19 \%[\mathrm{FD}=16.992]$ & $94.21 \%(8)^{\mathbf{0}}[\mathrm{FD}=\mathbf{1 7 . 0 6 1}]$ \\
\hline 2. & $\mathrm{~T} 4[28]$ & $98.59 \%$ & $88.11 \%[F D=18.012]$ & $\mathbf{9 4 . 4 6 \%}(18)[\mathrm{FD}=\mathbf{4 1 . 2 0 7}]$ \\
\hline 3. & T5 [28] & $98.93 \%$ & $95.38 \%[\mathrm{FD}=\mathbf{3 3 . 0 5 2}]$ & $95.69 \%(12)[F D=\mathbf{3 3 . 9 3 5}]$ \\
\hline 4. & T6 [28] & $97.23 \%$ & $86.04 \%[F D=8.91]$ & $\mathbf{9 2 . 5 2 \%}(27)[F D=\mathbf{2 8 . 8 0 8}]$ \\
\hline 5. & $\mathrm{~T} 7[28]$ & $98.91 \%$ & $95.73 \%[\mathrm{FD}=\mathbf{5 . 7 0 6 4}]$ & $95.67 \%(6)[F D=\mathbf{5 . 7 0 9 9}]$ \\
\hline 6. & $\mathrm{~T} 8$ [28] & $98.11 \%$ & $72.39 \%[F D=4.681]$ & $\mathbf{9 5 . 8 9 \%}(27)[F D=\mathbf{3 1 . 7 0 5}]$ \\
\hline 7. & T9 [28] & $95.14 \%$ & $96.73 \%[\mathrm{FD}=\mathbf{1 0 . 3 9 7}]$ & $96.73 \%(10)[F D=10.405]$ \\
\hline
\end{tabular}

Table 5: Results Comparison with Previous Publications 
Note: ${ }^{\mathbf{n}}$ MIRGS stands for multivariate iterative region growing using semantics algorithm in [28].

Note: ${ }^{\circ}$ Number in parenthesis indicates iterations of MFLD.

Table 5 and Table 6 show segmentation results in the previously published work in $[5,28]$ and those obtained using the proposed modified algorithm.

As an example, let us consider the results of texture T8 mentioned in Table 5. It is a complex 4-segment texture with straight boundaries from Brodatz texture database. The Fisher distance is 4.681 at $5^{\text {th }}$ iteration yielding accuracy of $72.39 \%$ and 31.433 at $27^{\text {th }}$ iteration yielding accuracy of $95.76 \%$. The accuracy is increased by $23.36 \%$. Similarly, the accuracy is increased for textures T4 and T6 relative to $5^{\text {th }}$ iteration as shown in Table 5.

\begin{tabular}{|c|c|c|c|c|}
\hline $\begin{array}{l}\text { Sr. } \\
\text { No. }\end{array}$ & Image name & $\begin{array}{l}\text { Accuracy with } \mathrm{MR}^{\mathrm{p}} \\
\text { algorithm [5] }\end{array}$ & $\begin{array}{c}\text { Accuracy with } \\
\text { MIRGS }^{\mathrm{n}} \text { algorithm } \\
{[28]}\end{array}$ & $\begin{array}{c}\text { Accuracy with the } \\
\text { proposed modification }\end{array}$ \\
\hline 1. & $\begin{array}{l}\text { D4-D77-D57-D84 } \\
\text { (4-segment Brodatz texture } \\
\text { with straight boundaries) }\end{array}$ & $\begin{array}{l}95.3 \%(5)^{\mathrm{s}} \\
{[\mathrm{FD}=\mathbf{6 . 3 9 6 8}]}\end{array}$ & --- & $\begin{array}{c}94.87 \%(10)^{\mathrm{q}} \\
{[\mathrm{FD}=\mathbf{7 . 9 6 2 1}]}\end{array}$ \\
\hline 2. & $\begin{array}{l}\mathrm{T} 1^{\mathrm{r}}[5,24,28] \\
\text { Mao-Jain Image }\end{array}$ & $\begin{array}{c}95.1 \%(5) \\
{[\mathrm{FD}=\mathbf{2 . 3 9 9 5}]}\end{array}$ & $98.40 \%$ & $\begin{array}{c}96.37 \%(7) \\
{[\mathrm{FD}=\mathbf{2 . 4 4 2 6}]}\end{array}$ \\
\hline 3. & $\begin{array}{l}\text { D4-D77 } \\
\text { (Two segment Brodatz texture } \\
\text { with sinusoidal boundary) }\end{array}$ & $\begin{array}{c}96.4 \%(5) \\
{[\mathrm{FD}=\mathbf{1 . 9 1 3 6}]}\end{array}$ & --- & $\begin{array}{c}97.11 \%(22) \\
{[\mathrm{FD}=1.9491]}\end{array}$ \\
\hline 4. & $\begin{array}{l}\mathrm{T}^{\mathrm{r}}[5,20,28] \\
\text { (Jain-Farrokhnia Image) }\end{array}$ & $\begin{array}{c}94.7 \%(5) \\
{[\mathrm{FD}=\mathbf{1 . 1 2 1}]}\end{array}$ & $96.32 \%$ & $\begin{array}{c}95.67 \%(10) \\
{[\mathrm{FD}=1.7043]}\end{array}$ \\
\hline
\end{tabular}

Table 6: Results Comparison with Previous Publications

Note:" MIRGS stands for multivariate iterative region growing using semantics algorithm in [28].

Note: ${ }^{p}$ MR stands for mixture resolving semi-supervised algorithm in [5].

Note: ${ }^{q}$ Number in parenthesis indicates iterations of MFLD.

Note: ${ }^{\mathbf{r}} \mathrm{T} 1$ is 4-class Brodatz texture image in [28]. T3 is 5-class Brodatz texture image in [28].

Note: ${ }^{\text {N }}$ Number in parenthesis indicates iterations of FLD in [5].

Table 5 shows that segmentation accuracies obtained using MIRGS algorithm in [28] are not much higher than those achieved by a modification deployed in [5]. The MIRGS algorithm is computationally very expensive and it is developed for complex textures. Therefore, the proposed modification is a reasonable alternative to it. Table 6 shows that the modified algorithm achieves slightly better results for the last three textures because the increase in Fisher distance is not significant. The modified algorithm in this study is tested on several hundred textures from five texture databases stated in the previous section. It is concluded, after rigorous testing, that the segmentation accuracy increased as high as $29.83 \%$ with iterations if Fisher distance was increased for highly complex textures and the combination of homogeneous and complex textures. To the best of our knowledge, it is nowhere stated in the relevant literature that increasing iterations of MFLD improves the localization of texture boundaries and segmentation accuracy of highly complex textures or a combination of complex and homogeneous textures.

\section{Conclusions and Future Scope}

The textures with straight boundaries and varying sinusoidal boundaries in the terms of different amplitude and different cycles over the edges of the boundaries are used for testing the performance of the proposed algorithm and boundaries of the textures are nicely detected as detailed in experimentation and results section. The proposed algorithm achieves the second rank in ten state-of-the-art algorithms on 21 images in Prague texture segmentation benchmark set. It is observed that texture segmentation accuracy significantly improves for complex fine textures and combination of homogeneous and complex fine textures when the number of iterations of multiclass Fisher linear discriminant (MFLD) is increased. The increased iterations of MFLD result into improved covariance matrices and hence optimum projection vectors which lead to a better segmentation solution. It can thus be inferred that the increasing number of iterations of MFLD results into an improvement in the localization of boundaries and segmentation accuracy of highly complex textures with small texture primitives with respect to the size of an image. The optimum texture segmentation results are obtained with both Gabor Filter and Gaussian smoothing of Gabor filter outputs implemented in frequency domain. Highly complex textures are segmented using spatial co-ordinates of pixels as texture features. Hence, the proposed algorithm is useful for texture images in which the same texture segments do not repeat and appear at different places in the input image.

Further improvement in accuracy can be achieved by performing adaptive smoothing of Gabor filter outputs. K-means clustering algorithm sometimes produces erroneous results and can be replaced by Equitz algorithm to avoid such fallacies. 


\section{References}

[1] Bello MG. A combined Markov random field and wave-packet transform-based approach for image segmentation. IEEE Trans. Image Process, 3(6), pp. 834-846, 1994.

[2] Bergen JR, Adelson EH. Early vision and texture perception. Nature, 333(26), pp. 363-364, 1988.

[3] Bigun J, Buf JM Du. N-folded symmetries by complex moments in Gaborian space and their application to unsupervised segmentation. IEEE Trans. Pattern Anal. Machine Intell, 16(1), pp. 80-87, 1994.

[4] Bovik AC, Clark M et al. Multichannel texture analysis using localized spatial filters. IEEE Trans. Patt. Anal. Mach. Int., 12(1), pp. 55-73, 1990.

[5] Clausi DA. K-means Iterative Fisher (KIF) unsupervised clustering algorithm applied to image texture segmentation. Pattern Recognition, 35(9), pp. 1959-1972, 2002.

[6] Clausi DA, Jernigan ME. Designing Gabor filters for optimal texture separability. Pattern Recognition, 33(11), pp. 1835-1849, 2000.

[7] Deng H and Clausi DA. Unsupervised image segmentation using a simple MRF model with a new implementation scheme. Pattern Recognition, 37(12), pp. 2323-2335, 2004.

[8] Duda RO, Hart PE, Pattern Classification. Wiley India. 2012, Ch.3, pp. 117-124.

[9] Dunn D, Higgins WE. Optimal Gabor filter for texture segmentation. IEEE Trans. Image Process, 4(7), pp. 947-964, 1995.

[10] Dunn D, Higgins WE, et al. Texture segmentation using 2-D Gabor elementary functions. IEEE Trans. Patt. Anal. Mach. Int., 16(2), pp. 130-149, 1994.

[11] Fukunaga K, Introduction to Statistical Pattern Recognition. Academic Press Inc. Toronto. 1990, Ch.4, pp. 131-152.

[12] Gonzalez Rafael C, Woods Richard E, Digital Image Processing. Third Edition Pearson Education. 2009, Ch.10, pp. 711-806.

[13] Gose, Johnsonbaugh et al, Pattern Recognition and Image Analysis. Prentice Hall India Publishers. 2015, Ch.37, pp. 329-418.

[14] Haindl M., Mike S, “Texture segmentation benchmark," in proc. IEEE Int. Conf. Pattern Recognition (ICPR). Dec.2008.

[15] Haralick RM, Shanmugan K et al. Textural features for image classification. IEEE Trans Syst, Man. Cybern, SMC3(6), pp. 610- 621, 1973.

[16] Hall CF, Hall EL. A nonlinear model for the spatial characteristics of the human visual system. IEEE Trans. Systems Man Cybern, SMC-7(3), pp. 161-170, 1977.

[17] Hoover Adam, Jean-Baptiste Gillian et al. An experimental comparison of range image segmentation algorithms. IEEE Transaction on Pattern Analysis and Machine Intelligence, 18(7), pp. 673-689, July 1996.

[18] Hossain Shahera, Serikawa Seiichi. Texture databases - A comprehensive survey. Pattern Recognition Letters, 34, pp. 2007-2022, 2013.

[19] Jain AK, Dubes RC, Algorithms for clustering Data. Prentice-Hall Englewood Cliffs, New Jersey. 1998, Ch.3, pp. 55-132.

[20] Jain AK, Farrokhnia F. Unsupervised texture segmentation using Gabor filters Pattern Recognitio, 24(12), pp. 1167$1186,1991$.

[21] Kervrann C, Heitz F. A Markov random field model-based approach to unsupervised texture segmentation using local and global spatial statistics. IEEE Trans Image Process, 4(6), pp. 856-862, 1995.

[22] Kiechle M, Storath M et al. Model-based learning of local image features for unsupervised texture segmentation. IEEE Transactions on Image Processing, 27(4), pp. 1994-2007, April 2018.

[23] Manjunath BS, Chellappa R. Unsupervised texture segmentation using Markov random field models. IEEE Trans. Patt. Anal. Mach. Int., 13(5), pp. 478-482, 1991.

[24] Mao J, Jain AK. A self-organizing network for hyper-ellipsoidal clustering (HEC). IEEE Trans Neural Networks, 7(1), pp. 16-29, 1996.

[25] Panda R, Chatterji BN. Unsupervised texture segmentation using tuned filters in Gaborian space. Pattern Recognition Letters, 13, pp. 445-453, 1997.

[26] Papoulis, Probability, Random Variables and stochastic Processes. New York: Dover, 1966, Ch.1-7, pp. 3-302.

[27] Pereyra M and McLaughlin S. Fast Unsupervised Bayesian Image Segmentation with Adaptive Spatial Regularisation. IEEE Trans. on Image processing, 30(6), pp. 2577- 2587, June 2017.

[28] Qin AK. and Clausi DA. Multivariate image segmentation using semantic region growing with adaptive edge penalty. IEEE Trans. Image Process, 8(19), pp. 2157-2170, August 2010.

[29] Randen T, Husoy JH. Filtering for texture classification: A comparative study. IEEE Trans. Patt. Anal. Mach. Int., 21(4), pp. 291- 310, 1999.

[30] Sonka M, Hlavac V et al, Image Processing, Analysis and Machine Vision. Second Edition-Brooks Publishing Company, 1993, Ch. 5-6, pp: 142-148, 237-239, 1999.

[31] Teuner Andreas, Pichler Olaf et al. Unsupervised Texture Segmentation of Images Using tuned Matched Gabor Filters. IEEE Trans. on image processing, 4(6), pp. 863-870, June 1995.

[32] Tomita Fumiaki and Tsuji Saburo, Computer Analysis of Visual Textures. Kluwer academic publishers, 1990, Ch.2,5,9, pp. 13-55, 71-82, 137-162 
[33] Yu Q and Clausi DA. SAR sea-ice image analysis based on iterative region growing using semantics. IEEE Trans. Geosci. Remote Sens, 45(12), pp. 3919-3931, Dec. 2007.

[34] Yu Q and Clausi DA. IRGS: Image segmentation using edge penalties and region growing. IEEE Trans Pattern Anal Mach Intell., 30(12), pp. 2126-2139, Dec. 2008.

[35] Yu Q and Clausi DA, "Combining local and global features for image segmentation using iterative classification and region merging," Proc. Second Canadian Conf. Computer and Robot Vision. Publisher: IEEE, May 2005.

[36] Zoltan Kato, Pong TC et al. Color image segmentation and parameter estimation in a markovian frame work. Pattern Recognition Letters, 22(3), pp. 309-321, 2001. 